\title{
Application of Linked Stress Release Model to Historical Earthquake Data: Comparison between Two Kinds of Tectonic Seismicity
}

\author{
Chunsheng Lu ${ }^{1,2}$ and DAVID Vere-Jones ${ }^{1}$
}

\begin{abstract}
The linked stress release model, incorporating a slow buildup of stress within a seismic region, its stochastic release through earthquakes and transfer between seismic regions, is applied to fit historical data from two typical kinds of seismicity: earthquakes occurring in intraplate (North China) and plate boundary (New Zealand) regions. The best model among different modifications of the basic model, which may reflect on a possible geophysical mechanism for earthquake occurrences, is obtained in terms of Akaike information criterion. For both tectonic regions studied, the linked stress release model fits the New Zealand data better than a collection of independent simple models, but is nearly indistinguishable from the simple stress release model in the case of North China. The seismicity in a plate boundary region due to subduction is more active and complex than that in an intraplate region due to collision between tectonic plates. The results highlight the major differences in tectonic seismicity, especially the heterogeneities of tectonic stress fields, and dynamic triggering mechanism with evidence that the crust may lie in a near-critical state.
\end{abstract}

Key words: Historical seismicity, tectonic stress fields, stress release, stress transfer, spatial interaction, triggering mechanism.

\section{Introduction}

Based on field observation and crustal deformation measurement of the 1906 San Francisco earthquake, REID (1911) proposed an elastic rebound theory of earthquake origins. According to the theory, stress in a seismically active region accumulates due to relative movement of faults. When the stress exceeds a certain threshold, for example the strength of rock media, an earthquake occurs and the accumulated strain energy is released in the form of seismic waves. Although this model and its modifications (so-called time- and slip-predictable models by fixing only strength or residual stress) have been widely used in long-term prediction (ShimAZAKI and NAKATA, 1980), real sequences of large earthquakes are fundamentally more complicated. We cannot exactly determine, at least at present, the

\footnotetext{
${ }^{1}$ School of Mathematical and Computing Sciences, Victoria University of Wellington, Wellington 600, New Zealand. E-mails: cslu@mcs.vuw.ac.nz, dvj@mcs.vuw.ac.nz

${ }^{2}$ LNM, Institute of Mechanics, Academia Sinica, Beijing 100080, China.
} 
strength of rock media in a seismic region. In other words, what we may know is that the risk of an earthquake occurrence will increase as the stress accumulates. Through a development of the Markov model suggested by KNOPOFF (1971), the stress release model, a stochastic version of elastic rebound theory, has been developed. It incorporates a deterministic buildup of stress within a region and its stochastic release through earthquakes, and has been applied to the statistical analysis of historical earthquake data in China, Japan and Iran (VERE-Jones, 1978; Vere-Jones and Deng, 1988; Zheng and Vere-Jones, 1991, 1994).

All the models based on elastic rebound theory suggest that a large earthquake should be followed by a period of quiescence, whereas in reality a strong earthquake can be followed by a period of activation and sometimes other earthquakes of comparable magnitude (GABRIELOv and Newman, 1994). This seems most plausibly consistent with a view of the events taking place in the earth's crust as forming part of a tightly linked, near-critical process, exhibiting the self-similarity, long-range correlation and power-law distributions which are characteristic of a physical process in near-critical state (VERE-JONES, 1976; TAKAYASU and MATSUZAKI, 1988; TurcotTe, 1992; MAIN, 1996; JAUMÉ and Sykes, 1999). Here, it is indeed observed that large events in one part of a region are noticeably often followed by large events in other quite distant parts of the region. One of the possible mechanisms is the competition between local strengthening and weakening through transfer and interaction of tectonic stress, these effects combining to cause a triggering mechanism of earthquake occurrences.

Although the stress field within a seismic region can be extracted from different kinds of information, the temporal variations of seismicity or historical earthquake data may most directly reflect the nature of earthquake-generating stress (ZHAO et al., 1990). In this paper we will use the linked stress release model, a natural extension of the simple stress release model incorporating the stress transfer among subregions, to interpret the historical earthquake catalogues from China and New Zealand. The results obtained underline the major differences in tectonic structures and earthquake mechanisms between the two regions.

\section{Linked Stress Release Model}

In the univariate stress release model, the regional stress level $X(t)$ increases deterministically between two earthquakes and releases stochastically as a scalar Markov process. The evolution of stress versus time is assumed to follow the equation

$$
X(t)=X(0)+\rho t-S(t)
$$

where $X(0)$ is the initial stress level, $\rho$ is the constant loading rate from external tectonic forces, and $S(t)=\Sigma_{t_{i}<t} S_{i}$, where $t_{i}, S_{i}$ are the origin time and stress release 
associated with the $i$-th event (Vere-Jones and Deng, 1988; Zheng and VereJONES, 1991, 1994).

Obviously, issues of stress readjustment and transfer cannot be considered in this simple framework. In order to take into account interactions among different subregions due to stress transfer, the evolution of stress $X_{i}(t)$ in the $i$-th subregion versus time is rewritten as

$$
X(t, i)=X(0, i)+\rho_{i} t-\sum_{j} \theta_{i j} S(t, j)
$$

where $S(t, j)$ is the accumulated stress release from events within the subregion $j$ over the period $(0, t)$, and the coefficient $\theta_{i j}$ measures the fixed proportion of stress drop from events, initiated in the subregion $j$, which is transferred to the subregion $i$. Here, $\theta_{i j}$ may be positive or negative, resulting in damping or excitation respectively. It is convenient, if ignoring aftershocks, to set $\theta_{i i}=1$ for all $i$. We call this new version a linked (or coupled) stress release model (SHI et al., 1998; LIU et al. 1998; LU et al., 1999a,b,c). If $\theta_{i j}=0$ for all $i \neq j$ in (2), the model is reduced to an independent combination of simple forms as in (1).

The value of stress release during an earthquake can be estimated from its magnitude in terms of the relation $M=\frac{2}{3} \log _{10} E+$ const (KANAMORI and ANDERSON, 1975), where $E$ is the released energy during an earthquake. For simplicity, the stress drop $S$ during an earthquake is presumed to be proportional to the square root of the released energy, i.e., $S \propto E^{1 / 2}$. Then, we have the formula

$$
S=10^{0.75\left(M-M_{0}\right)}
$$

where $M_{0}$ is the threshold magnitude.

The probability intensity of an earthquake occurrence is controlled by a risk function $\Psi(x)$. Generally speaking, the risk function should be increasing nonlinearly with the stress level $x$. If the solid media had an exact critical strength, $\Psi(x)$ would have to be zero until $x$ reached the critical strength, and infinite beyond it. By contrast, a finite constant value of $\Psi(x)$ corresponds to a pure random process in which the occurrence of events is independent of the stress level. Thus, a simple choice of $\Psi(x)$ is the exponential function $\Psi(x)=\exp (\mu+v x)$, where $\mu$ and $v$ are constants and indicate the background and sensitivity to risk, respectively. This is a convenient compromise between time-predictable and purely random (Poisson) processes.

It is further assumed that the probability distribution of earthquake sizes is independent of stress level (and as a default governed by the standard GutenbergRichter law). The key for statistical analysis is that the data in historical earthquake catalogues can be treated as a marked point process in time-stress space with the conditional intensity function 


$$
\lambda(t, i)=\exp \left\{a_{i}+v_{i}\left[\rho_{i} t-\sum_{j} \theta_{i j} S(t, j)\right]\right\}
$$

where $\alpha_{i}=\mu_{i}+v_{i} X_{i}(0), v_{i}, \rho_{i}$ and $\theta_{i j}$ are the parameters to be fitted. We choose to parameterise the intensity in this form because it is more amenable to physical intuition (i.e., the $\rho_{i}$ are input rates and the $v_{i}$ are sensitivities to increase in stress). A simpler parameterisation can be recovered by setting $b_{i}=v_{i} \rho_{i}, c_{i j}=\theta_{i j} /$ $\rho_{i}$. Estimates of the parameters are found by maximising the log-likelihood

$$
\log L=\sum_{i}\left[\sum_{j} \log \lambda\left(t_{j}, i\right)-\int_{T_{1}}^{T_{2}} \lambda(t, i) d t\right]
$$

where the observation interval $\left(T_{1}, T_{2}\right)$ contains events at times $T_{1}<t_{j}<T_{2}$ ( $j=$ $1,2, \ldots, N)$, and $N$ is the number of events. This can be done numerically by using routines in the statistical seismology library (SSLib) developed recently (HARTE, 1998).

With discretion in the degree of interaction and subregions, we have a number of possible models. The choice among these models will be based on the Akaike information criterion (AIC), which is defined as

$$
\mathrm{AIC}=-2 \log \hat{L}+2 k
$$

where $\log \hat{L}$ is the maximum $\log$-likelihood for a given model and $k$ is the number of parameters to be fitted in the model (AKAIKE, 1977). This represents a rough way of compensating for the effect of adding parameters, and is a useful heuristic measure of the relative effectiveness of different models, in avoiding overfitting. For example, the simple stress release model $\left(\theta_{i j}=0\right.$ for $i \neq j$ in (4)) with three parameters as against the Poisson model with only one $\left(\alpha_{i}\right)$ or the Poisson with exponential trend with two $\left(\alpha_{i}, \beta_{i}=\rho_{i} v_{i}\right)$, must demonstrate a significantly better fit to justify the additional parameters. In typical cases, model differences which would be significant at around the $5 \%$ confidence level correspond to differences in AIC values of around 1.5 or 2 . The best model is that for which AIC has the smallest value. However, we should caution that the AIC values obtained here should be used as rough guides only, since the amount of historical earthquake data is not too large and the distribution of the log-likelihood is nonstandard (DALEY and VERE-JONES, 1988).

Using different combinations of the parameters in (4), we can examine different stress interaction mechanisms. If we assume all the parameters $\theta_{i j} \neq 0$, longrange interaction is allowed; otherwise, if we only let $\theta_{i j} \neq 0$ for neighbouring regions, short-range interaction will predominate. On the other hand, special combinations of these parameters, such as the risk level $v_{i}$, the loading rate $\rho_{i}$ etc., can be used to identify tectonic features and difference between different tectonic seismicity. 


\section{Applications to Historical Catalogues}

Several versions of the linked stress release model have been implemented to both the historical earthquake catalogues and synthetic ones generated by geophysical models (SHI et al., 1998; LiU et al., 1998; Iмото et al., 1999; Lu et al., 1999a,b,c). When we analyse the real data, considerable caution should be taken to the completeness of historical catalogues used in the modelling. Here, the data require expert scrutiny and advice in aspects such as the analysis and selection of individual events, choice of time and magnitude thresholds, interpretation of magnitudes and epicentres, etc. We also need to stress that in all the data sets studied, aftershocks should be deliberately excluded from the catalogues since the model framework presented above is only suitable to the analysis of mainshocks (UTSU et al., 1995). Fortunately, since the data in the two cases to be studied are restricted to large events, the numbers of aftershocks exceeding the threshold are usually very small, consequently their exclusion or inclusion should not have a major effect on the analysis.

\subsection{Chinese Data (North China)}

In general, the Chinese historical catalogue is one of the oldest and most extensive in existence. The region selected here represents a substantial part of northern China, and comprises the northeastern coastal region, excluding the Yellow Sea but including the Bohai Sea, and the main part of the Ordos Plateau, including in particular its western and southern boundaries. It is essentially bounded by latitudes $32^{\circ} \mathrm{N}$ and $42^{\circ} \mathrm{N}$, longitudes $104^{\circ} \mathrm{E}$ and $120^{\circ} \mathrm{E}$, and covers about the 500-year period 1480-present. The catalogue for this region is believed to be complete for events with $M \geq 6.0$ (GU, 1983; ZHENG and VERE-Jones, 1991). Here, $M_{0}=6.0$ is used as the lower threshold (see Figs. 1 and 2).

Geophysical considerations suggest that the region should be treated as containing at least two major components: the coastal region and the Ordos Plateau, roughly separated by the Taihung Mountains. To the east of this region the principle stress is compressive, oriented in the $\mathrm{E}-\mathrm{W}$ direction, and corresponds to pressure from the Pacific Plate. To its west, the stress is mainly in a $\mathrm{N}-\mathrm{S}$ direction and driven by pressure from the Indian Plate and Tibetan Plateau (Li and LiU, 1986; MA et al., 1990).

As illustrated in Figures 1 and 2, the subregions and data set used are the same as in ZHENG and Vere-Jones (1991, 1994), except that several current events have been added to the catalogue. The total number of events is 66,33 in the western part and 33 in the eastern part.

The three kinds of basic models (i.e., the Poisson model, the simple stress release model and linked stress release model), are applied to the analysis of each subregion. The results are set out in Table 1. In terms of the difference between 


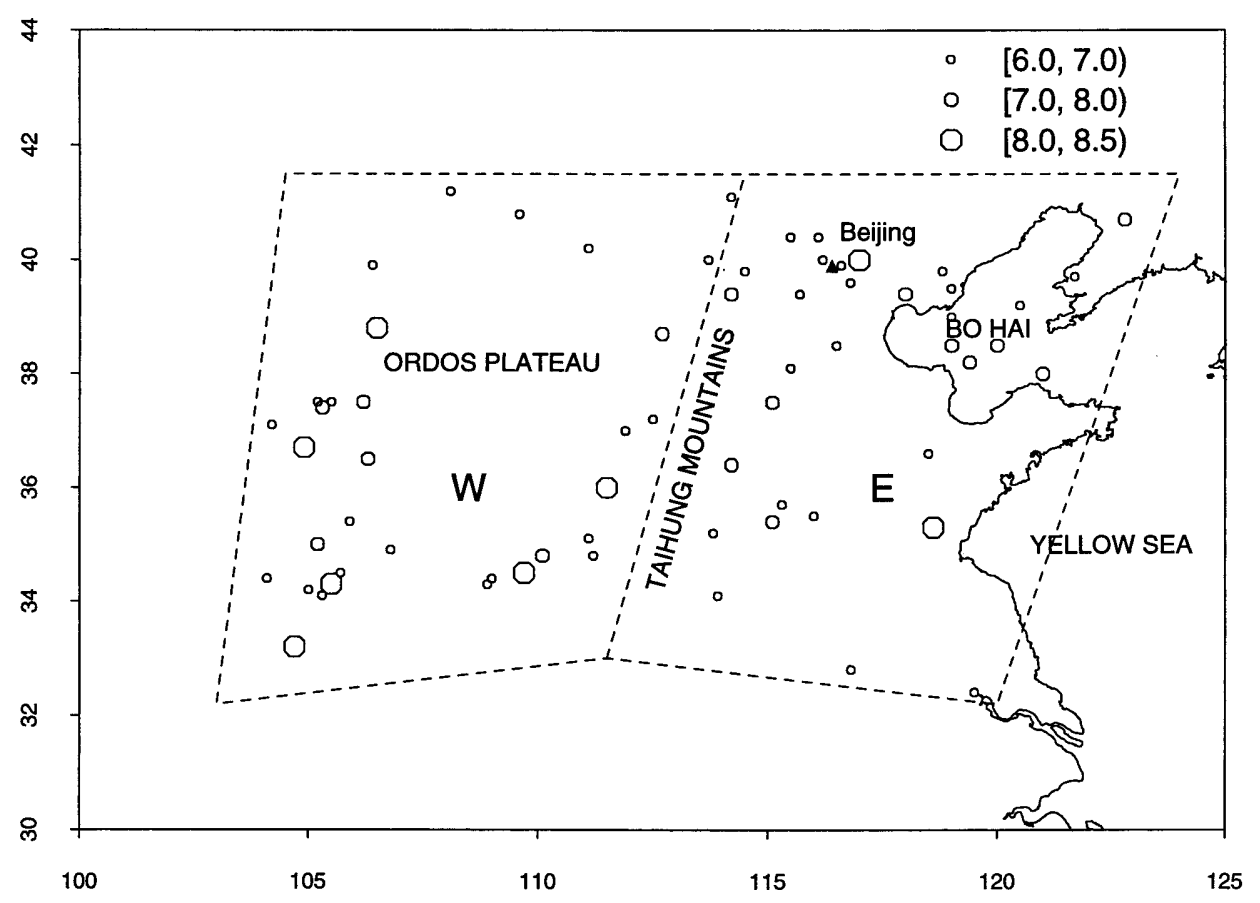

Figure 1

The epicentre distribution of major earthquakes with magnitude $M \geq 6.0$ and the definition of subregions of the North China historical earthquakes. The region is divided into two subregions: the western part (W) and the eastern part (E), respectively. The total number of events is 66,33 in the western part and 33 in the eastern part. Please note that the subregion boundaries (dashed line) are used only as an eye's guide.

AIC values, it is obvious that the simple stress release model fits the data better than the Poisson model. As discussed above, we can choose different combinations of the parameters in (4) to fit the data. Here, the best fitting model, having the smallest AIC value, is obtained when we let all the initial risk levels $\left(\alpha_{i}\right)$, the risk sensitivities $\left(v_{i}\right)$ and the loading rates $\left(\rho_{i}\right)$ be equal for both subregions. In fact, this is a natural choice if we compare the parameters fitted by simple stress release models. Although the difference between the AIC values is too small to clearly indicate a preferred model, the similarity in the parameter values for the two regions, and the fact that fewer parameters $(k=5)$ are needed in the linked stress release model, suggest that the latter model provides the more effective description of the data. Thus it seems that northern China can be viewed as a single region with similar seismicity and modest interactions between two subregions.

The risk functions calculated by using the fitted parameters are shown in Figure 3. The negative value of $\theta_{12}$ implies that events occurring in subregion $E$ tend to trigger events in subregion $W$. Therefore, the main compressive stress may be in the 


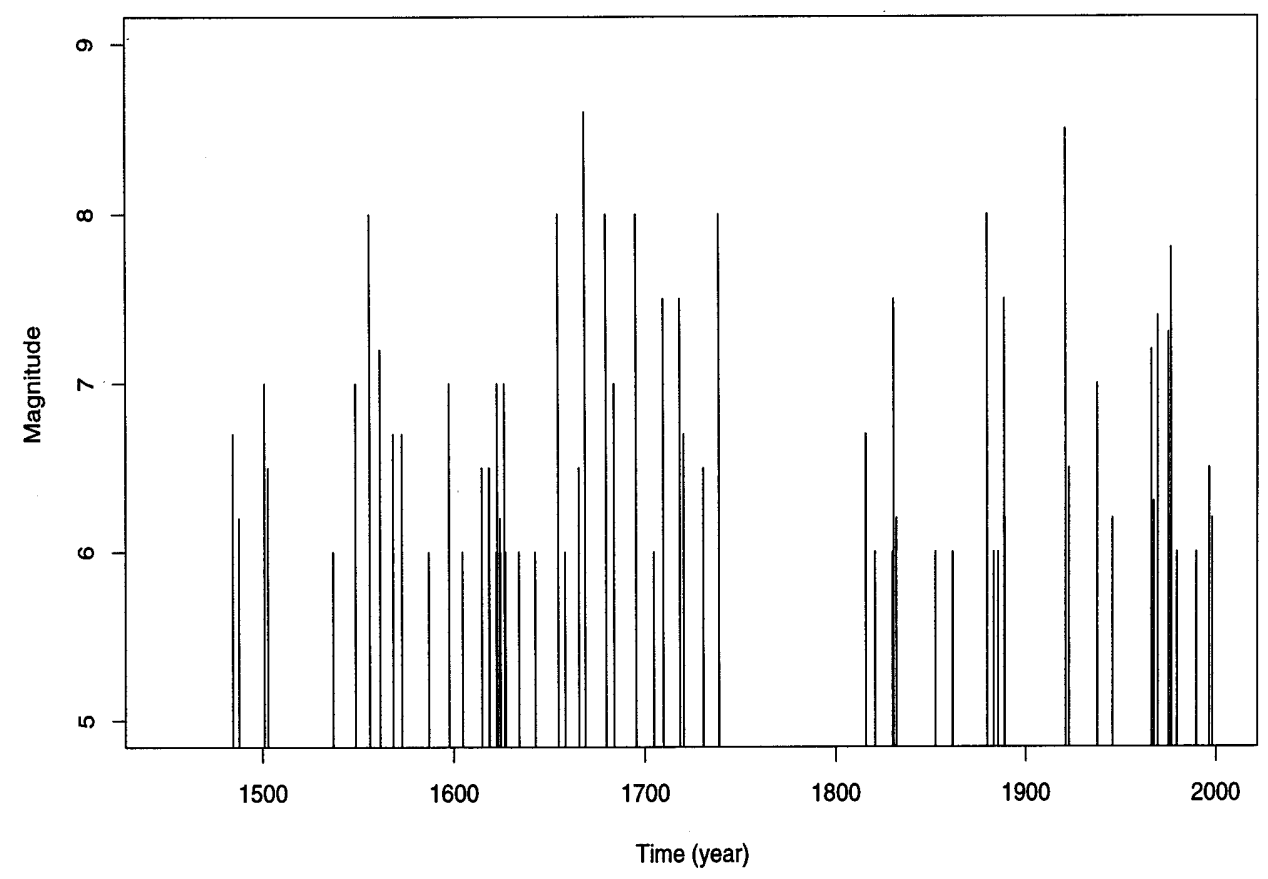

Figure 2

The magnitude versus time of the North China historical earthquakes with magnitude $M \geq 6.0$ during the period from 1480 to 1998.

Table 1

The fitted parameters and AIC values obtained by using the Poisson model, the simple stress release model (SRM) and the linked stress release model (LSRM) to the North China historical earthquake data

\begin{tabular}{lllllllll}
\hline Model & Subregion $i$ & $\alpha_{i}$ & $v_{i}$ & $\rho_{i}$ & $\theta_{i 1}$ & $\theta_{i 2}$ & $k$ & AIC \\
\hline Poisson & 1 (W) & -2.755 & & & & & 2 & 499.72 \\
& 2 (E) & -2.755 & & & & & & \\
SRM & 1 (W) & -3.192 & 0.0042 & 3.599 & & & 6 & 490.24 \\
& 2 (E) & -3.457 & 0.0043 & 3.031 & & & & \\
LSRM & 1 (W) & -3.273 & 0.0042 & 3.033 & 1.000 & -0.231 & 5 & 488.96 \\
& 2 (E) & -3.273 & 0.0042 & 3.033 & 0.025 & 1.000 & & \\
\hline
\end{tabular}

E-W direction, in agreement with the conclusion from the viewpoint of plate tectonics (Li and LiU, 1986).

\subsection{New Zealand Data}

Compared to the Chinese historical catalogue, the New Zealand catalogue is relatively incomplete, and has a short record history. Although the main upgrade of 
Subregion W
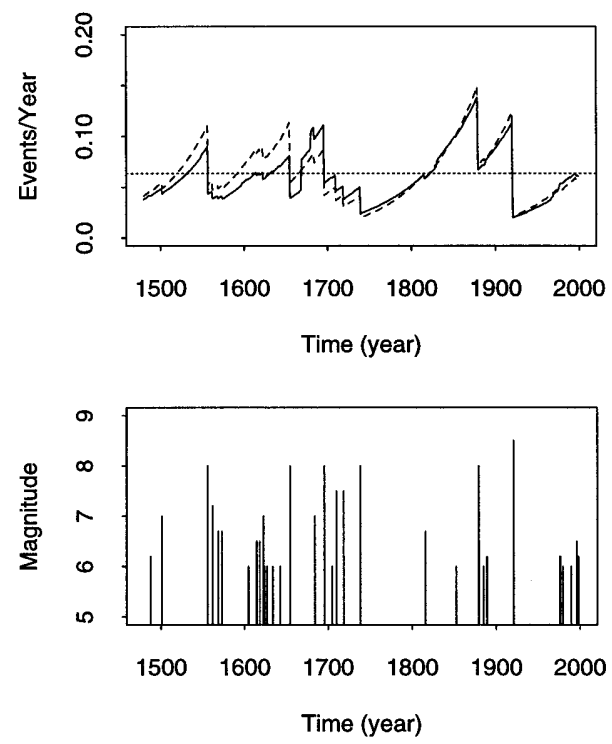

Subregion $\mathrm{E}$
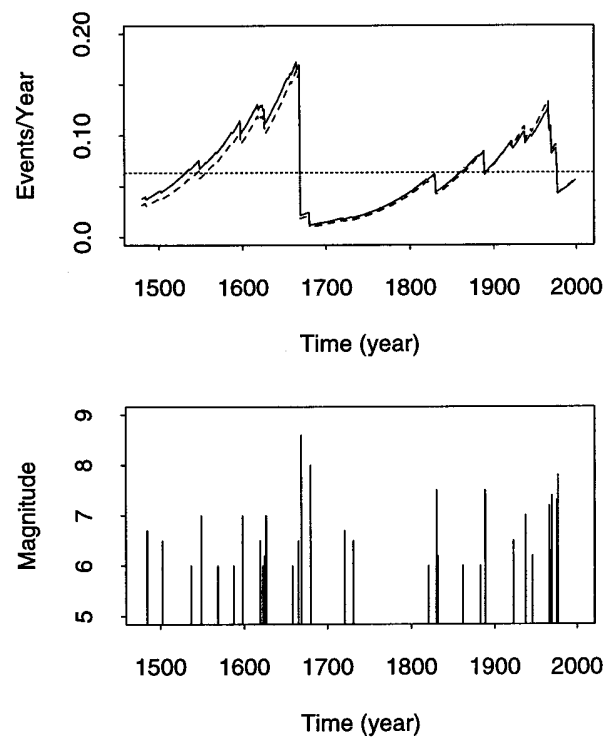

Figure 3

The risk function (events/year) versus time (year) for each subregion calculated by the linked stress release model (solid line), the simple stress release model (dashed line), and the Poisson model (dotted line). For comparison the earthquake versus time for each subregion is also plotted.

seismological recording in New Zealand occurred in the late 1980s (ANDERSON and WEBB, 1994), the catalogue with the magnitude $M \geq 6.0$ should be reasonably complete for shallow events since 1840. To decrease the chance of bias from the historical records, especially from the pre-instrumental earthquake catalogues, a lower threshold of $M_{0}=6.2$ has been used, as shown in Figures 4 and 5.

As an active island arc structure, New Zealand seems unlikely to consist of a few relatively well-defined seismic components, but rather of a closely interacting, highly fragmented ensemble within which a few larger units are embedded. The seismicity of the region studied here (see Fig. 4) is dominated by the boundaries of subducting zone of the Pacific plate beneath the Australian plate. The definition of appropriate subregions, which must satisfy both geophysical and statistical requirements, is in no case trivial. One way that might be used to define subregions is by the application of some clustering algorithm, with boundaries drawn equidistant between neighbouring clusters. An additional consideration is that subregions must include sufficient observations to allow the numerical parameter fitting procedure to converge. The choice of the two subregions $(\mathrm{N}$ and $\mathrm{S}$ ) provided in the present study, as illustrated in Figure 4, is not claimed to be optimal, or more exactly 


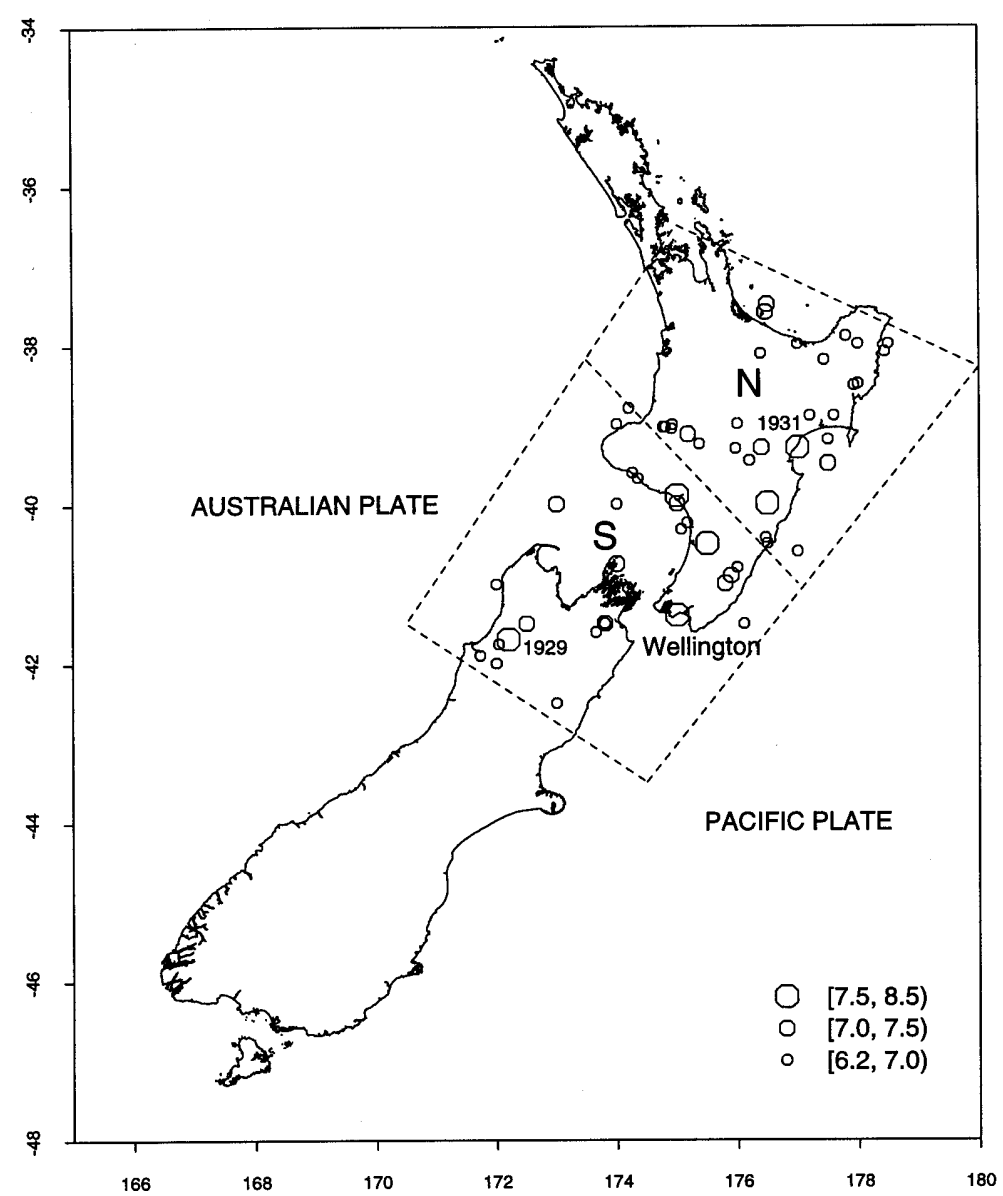

Figure 4

The epicentre distribution of major earthquakes with magnitude $M \geq 6.2$ and the definition of subregions of the New Zealand historical earthquakes. The region is divided into two subregions: the northern part $(\mathrm{N})$ and the southern part $(\mathrm{S})$, respectively. The total number of events is 65,31 in the northern part and 34 in the southern part. Please note that the subregion boundaries (dashed line) are used only as an eye's guide

speaking, is just a preliminary attempt. Here, the total number of events is 65,31 in the northern part and 34 in the southern part.

As with the analysis of the North China data, three basic models are applied to each subregion. The results are shown in Table 2. The simple stress release model for each subregion is an improvement on the Poisson model, and further substantial improvement is obtained by the linked stress release model in which the difference of the AIC values is about 8.80 or 9.2 , although more parameters $(k=8$ or $k=7)$ are needed in the latter. 


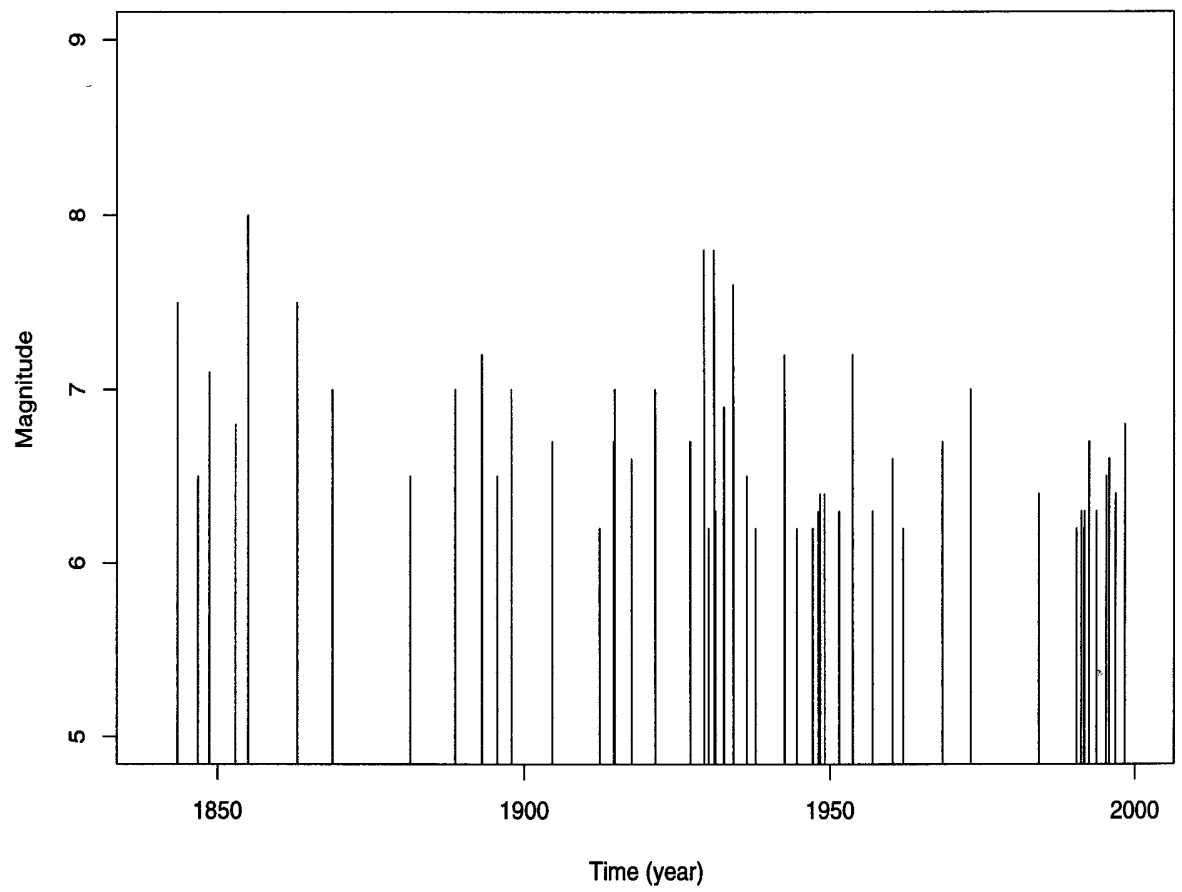

Figure 5

The magnitude versus time of the New Zealand historical earthquakes with magnitude $M \geq 6.2$ during the period spanning 1840 to 1998 .

Table 2

The fitted parameters and AIC values obtained by using the Poisson model, the simple stress release model (SRM) and the linked stress model (LSRM) to the New Zealand historical earthquake data, where CRLSRM represents the common loading rates introduced in the linked stress release model

\begin{tabular}{lllllllll}
\hline Model & Subregion $i$ & \multicolumn{1}{l}{$\alpha_{i}$} & $v_{i}$ & $\rho_{i}$ & $\theta_{i 1}$ & $\theta_{i 2}$ & $k$ & AIC \\
\hline Poisson & $1(\mathrm{~S})$ & -1.629 & & & & & 2 & 339.44 \\
& $2(\mathrm{~N})$ & -1.536 & & & & & & \\
SRM & $1(\mathrm{~S})$ & -1.132 & 0.0051 & 5.968 & & & 6 & 330.46 \\
& $2(\mathrm{~N})$ & -3.130 & 0.0026 & 10.391 & & & & \\
LSRM & $1(\mathrm{~S})$ & -0.896 & 0.0056 & 4.578 & 1.000 & -0.261 & 8 & 321.66 \\
& $2(\mathrm{~N})$ & -7.893 & 0.0127 & 0.772 & -1.179 & 1.000 & & \\
CRLSRM & $1(\mathrm{~S})$ & -0.851 & 0.0035 & 1.182 & 1.000 & -0.866 & 7 & 321.26 \\
& $2(\mathrm{~N})$ & -7.908 & 0.0129 & 1.182 & -1.110 & 1.000 & & \\
\hline
\end{tabular}

The risk functions calculated by using the fitted parameters are shown in Figure 6. The seismicity of the two subregions is very different. The risk in subregion $\mathrm{N}$ is higher than that in subregion $\mathrm{S}$. This is well consistent with the recent results 
Subregion S
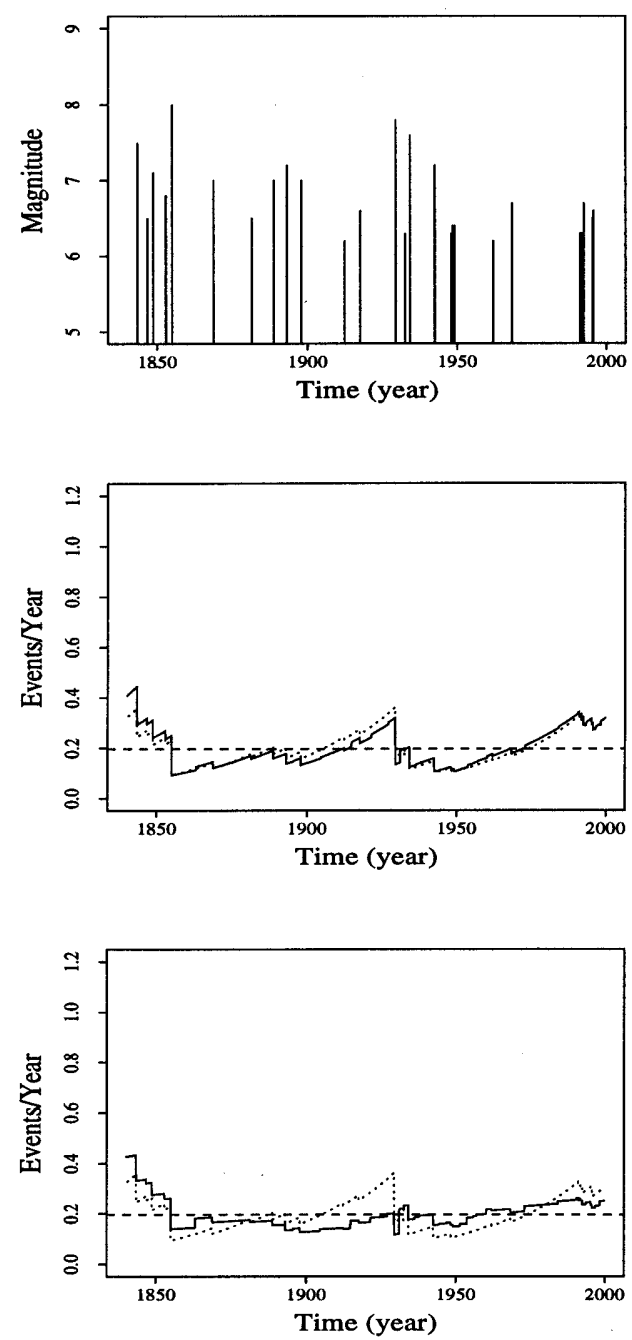

Subregion $\mathbf{N}$
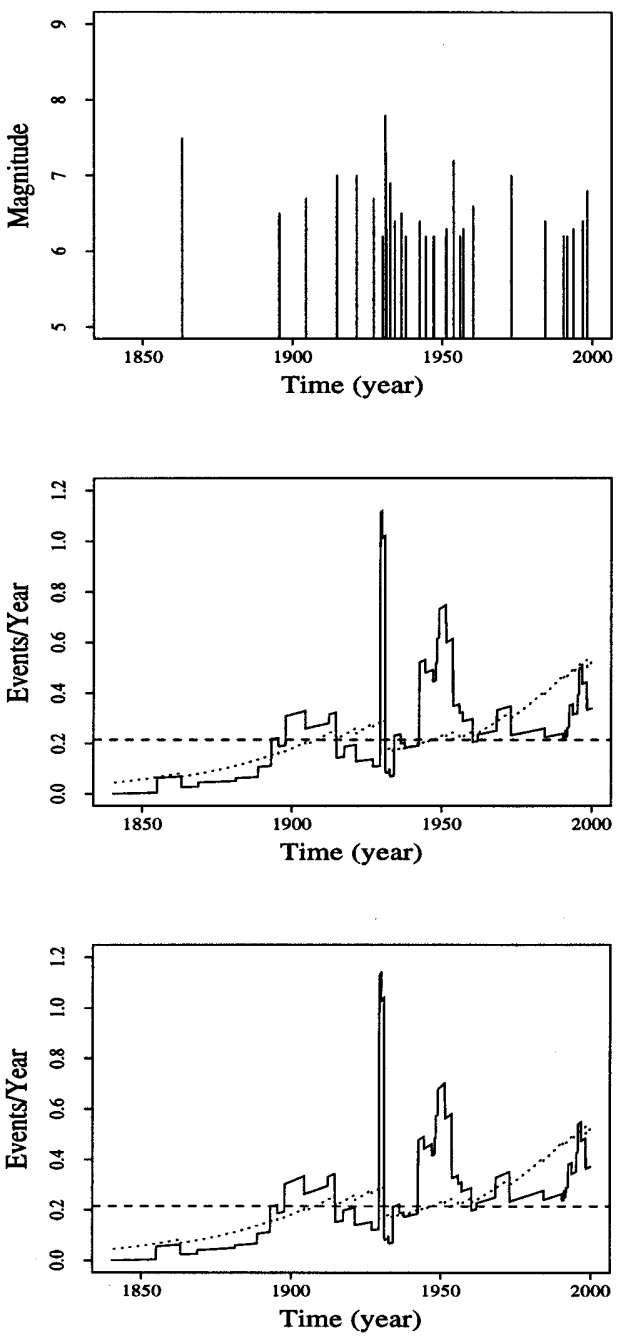

Figure 6

The risk function (events/year) versus time (year) for each subregion calculated by the linked stress release model (solid line) without (middle) or with (bottom) the loading rates $\rho_{i}$ being constrained to be equal, the simple stress release model (dashed line), and the Poisson model (dotted line). For comparison, the earthquake versus time for each subregion is also plotted.

discovered by the $M 8$ and MSc (i.e., the Mendocino Scenario) algorithms (KossOвокоv et al., 1999). Carefully checking the parameters in Table 2, we find both the interaction coefficients $\left(\theta_{21}\right.$ and $\left.\theta_{12}\right)$ are negative, which indicates that events occurring in one subregion will increase the risk and trigger events in the other subregion. In particular the very large value of $\theta_{21}$, coupled with the low value of 
$\rho_{2}$, suggests that a large part of the risk in the lower North Island is due to the triggering effect of events in the South Island. This feature is retained even in the third model considered, where (as in the study of Japanese data by LU et al. (1999a)) the loading rates $\rho_{i}$ are constrained to be equal. This results in a model with one less parameter and marginally better AIC than the unconstrained model. As shown in Figure 6, the Hawke's Bay earthquake $(M=7.8,2$ Feb., 1931) in the subregion $\mathrm{N}$ seems to be triggered by the Buller earthquake $(M=7.8,16$ June, 1929 ) in the subregion S (Fig. 4). Such triggering effects may be a common feature of subduction seismicity since similar behaviour was also discovered in our analysis of the Japanese historical data (Tokyo-Kamakura region) (Zheng and VereJONES, 1994; Lu et al., 1999a).

\section{Discussion and Conclusion}

In this paper, the linked stress release model, incorporating stress transfer and spatial interaction, is proposed and applied to the historical earthquake data from North China and New Zealand. The results show that in both cases studied, the linked stress release model fits the New Zealand data better than a collection of independent simple models, but is nearly indistinguishable from the simple stress release model in the case of North China. Moreover, the differences in the results for the two regions appear to reflect the differences in the tectonic settings of the regions. As we have seen, the seismicity in the plate boundary (subduction) region is more active and complex than that in the intraplate (plate collision) region. Thus more parameters (different loading rates, risk levels, etc.) in the models are needed for the description of the seismicity at plate boundary. As MATSU'URA and SATO (1997) pointed out that, for example, the assumption of uniform loading may not be appropriate for large interplate earthquakes.

In the linked stress release model proposed in this paper, the stress field is treated as a scalar which is representative of the whole region being studied. This is only likely to be a reasonable approximation if the real stress field has a dominant principal component which is not greatly variable, either in strength or direction, across the region. Theoretically speaking, it seems not to be difficult to introduce the stress field as a tensor in the framework of the model presented here, but the real data that can be used are not large enough for such a statistical analysis.

We should also note that the length scales in the two regions are substantially different. The dimension of one subregion in North China is approximately corresponding to the entire New Zealand region. In order to consider the possible scale-dependent effect, we further divided the North China region into four subregions: two in the western part and two in the eastern part (ZHENG and VERE-Jones, 1994). This gives us an opportunity to check whether there is a 
long-range stress interaction among subregions. The results are similar to those obtained above, i.e., no clearly preferred model emerges.

In summary, the results furnish a possible hint that the crust may lie in a near-critical state, so that an earthquake occurring in one region could trigger another earthquake distant from it within the region. The model itself provides a simple paradigm whereby spatio-temporal complexity of seismicity can be related to both the dynamics and heterogeneities in a seismic region (KAGAN, 1994; BENZION, 1996). Despite the crudity of the model in physical terms, it has the advantage of fitting simple physical ideas into a stochastic framework, thereby allowing it to be objectively fitted and tested on real data. On the other hand, we can also apply this model to the synthetic catalogues, especially to those generated by considering special tectonic regions (ROBINSON and BENITES, 1996) or geophysical mechanism (MORA and PlACE, 1998). Using the fitted parameters, we can forecast the long-term risk in a seismic region (see OGATA, 1981) and improve simulation models for earthquake process.

\section{Acknowledgements}

This work is supported by the Marsden Fund administered by the Royal Society of New Zealand. We would like to thank Y. Shi, X. Zheng, L. Ma, M. Bebbington, H. Takayasu, and especially D. Harte for many valuable discussions and assistance. The paper has benefited from the comments of two anonymous referees.

\section{REFERENCES}

Anderson, H., and Webb, T. (1994), New Zealand Seismicity: Patterns Revealed by the Upgraded National Seismograph Network, New Zeal. J. Geol. Geophys. 37, 477-493.

Akaike, H., On entropy maximisation principle. In Applications of Statistics (ed. Krishnaiah, P. R.) (North Holland, Amsterdam 1977) pp. 27-41.

Ben-Zion, Y. (1996), Stress, Slip, and Earthquakes in Models of Complex Single-fault Systems Incorporating Brittle and Creep Deformations, J. Geophys. Res. 101, 5677-5706.

Daley, D., and Vere-Jones, D., An Introduction to the Theory of Point Processes (Springer, Berlin 1988) $702 \mathrm{pp}$.

Gabrielov, A., and Newman, W. I., Seismicity modelling and earthquake prediction: A review. In Nonlinear Dynamics and Predictability of Geophysical Phenomena (eds. Newman, W. I., Gabrielov, A., and Turcotte, D. L.) (Am. Geophys. Union, Washington, D.C. 1994) pp. 7-13.

Gu, G. X., Chinese Earthquake Catalogue (in Chinese) (Science Press, Beijing 1983) 895 pp.

Harte, D. (1998), Documentation for the Statistical Seismology Library, Rep. Sch. Math. Comput. Sci., Victoria Univ. Wellington 10,1-101.

Imoto, M., Maeda, K., and Yoshida, A. (1999), Use of Statistical Models to Analyse Periodic Seismicity Observed for Clusters in the Kanto Region, Central Japan, Pure appl. geophys. 155, 609-624.

Jaumé, S. C., and Sykes, L. R. (1999), Evolving towards a Critical Point: A Review of Accelerating Seismic Moment/Energy Release Prior to Large and Great Earthquakes, Pure appl. geophys. 155, 279-306. 
Kagan, Y. Y. (1994), Observational Evidence for Earthquakes as a Nonlinear Dynamic Process, Physica D $77,160-192$.

Kanamori, H., and Anderson, D. L. (1975), Theoretical Basis of Some Empirical Relations in Seismology, Bull. Seismol. Soc. Amer. 65, 1073-1095.

Knopoff, L. (1971), A Stochastic Model for the Occurrence of Main-sequence Earthquakes, Rev. Geophys. Space Phys. 9, 175-188.

Kossobokov, V. G., Romashrova, L. L., Keilis-Borok, V. I., and Healy, J. H. (1999), Testing Earthquake Prediction Algorithms: Statistically Significant Advance Prediction of the Largest Earthquakes in the Circum-Pacific, 1992-1997, Phys. Earth Planet. Int. 111, 187-196.

LI, F. G., and Liu, G. X. (1986), Stress State in the Upper Crust of the China Mainland, J. Phys. Earth (Suppl.) 34, S71-S80.

Liu, J., Vere-Jones, D., Ma, L., Shi, Y., and Zhuang, J. (1998), The Principle of Coupled Stress Release Model and Its Application, Acta Seismol. Sinica 11, 273-281.

Lu, C., Harte, D., and Bebbington, M. (1999a), A Linked Stress Release Model for Historical Japanese Earthquakes: Coupling Among Major Seismic Regions, Earth Planets Space 51, 907-916.

Lu, C., Vere-Jones, D., and Takayasu, H. (1999b), Avalanche Behaviour and Statistical Properties in a Microcrack Coalescence Process, Phys. Rev. Lett. 82, 347-350.

Lu, C., Vere-Jones, D., Takayasu, H., Tretyakov, A. Yu., and Takayasu, M. (1999c), Spatiotemporal Seismicity in an Elastic Block Lattice Model, Fractals 7, 301-311.

Ma, Z., Fu, Z., Zhang, Y., Wang, C., Zhang, G., and Liu, D. Earthquake Prediction: Nine Major Earthquakes in China (1966-1976) (Seismological Press, Beijing 1990) 332 pp.

Main, I. (1996), Statistical Physics, Seismogenesis, and Seismic Hazard, Rev. Geophys. 34, 433-462.

Matsu'Ura, M., and Sato, T. (1997), Loading Mechanism and Scaling Relations of Large Interplate Earthquakes, Tectonophysics 277, 189-198.

Mora, P., and Place, D. (1998), Numerical Simulation of Earthquake Faults with Gauge: Towards a Comprehensive Explanation for the Heat Flow Paradox, J. Geophys. Res. 103, 21,067-21,089.

Ogata, Y. (1981), On Lewis's Simulation Method for Point Processes, IEEE Trans. Inf. Theory 27, $23-31$.

ReID, H. F. (1911), The Elastic-rebound Theory of Earthquakes, Univ. Calif. Publ. Geol. Sci. 6, 413-444.

Robinson, R., and Benites, R. (1996), Synthetic Seismicity Models for the Wellington Region, New Zealand: Implications for the Temporal Distribution of Large Events, J. Geophys. Res. 101, 27,83327,844 .

Shi, Y., Liu, J., Vere-Jones, D., Zhuang, J., and Ma, L. (1998), Application of Mechanical and Statistical Models to Study of Seismicity of Synthetic Earthquakes and the Prediction of Natural Ones, Acta Seismol. Sinica 11, 421-430.

Shimazaki, K., and Nakata, T. (1980), Time Predictable Recurrence Model for Large Earthquakes, Geophys. Res. Lett. 12, 717-719.

Takayasu, H., and Matsuzaki, M. (1988), Dynamical Phase Transition in Threshold Elements, Phys. Lett. A $131,244-247$.

Turcotte, D. L. Fractals and Chaos in Geology and Geophysics (Cambridge University Press, Cambridge 1992) $221 \mathrm{pp}$.

Utsu, T., Ogata, Y., and Matsu'ura, R. S. (1995), The Centenary of the Omori Formula for a Decay Law of Aftershock Activity, J. Phys. Earth 43, 1-33.

Vere-Jones, D. (1976), A Branching Model for Crack Propagation, Pure appl. geophys. 114, 711-726.

Vere-Jones, D. (1978), Earthquake Prediction: A Statistician's View, J. Phys. Earth 26, 129-146.

Vere-Jones, D, and Deng, Y. L. (1988), A Point Process Analysis of Historical Earthquakes from North China, Earthquake Res. China 2, 165-181.

Zhao, Z., Oike, K., Matsumura, K., and Ishikawa, Y. (1990), Stress Field in the Continental Part of China Derived from Temporal Variations of Seismic Activity, Tectonophysics 178, 357-372.

Zheng, X., and Vere-Jones, D. (1991), Application of Stress Release Models to Historical Earthquakes from North China, Pure appl. geophys. 135, 559-576.

Zheng, X., and Vere-Jones, D. (1994), Further Applications of the Stochastic Stress Release Model to Historical Earthquake Data, Tectonophysics 229, 101-121.

(Received August 7, 1999, revised March 13, 2000, accepted April 6, 2000) 